\title{
Oxidant-Antioxidant Status and c-myc Expression in BPA- and DEHP-Exposed Zebrafish Embryos
}

\author{
Unsal Veli Ustundag', Ismail Unal' ${ }^{2}$, Perihan Seda Ates ${ }^{2}$, Ahmet Ata Alturfan ${ }^{3}$, \\ Turkan Yigitbasi' ${ }^{1}$, Ebru Emekli Alturfan ${ }^{2 *}$
}

'Istanbul Medipol University, School of Medicine, Department of Biochemistry, Istanbul, Turkey

2Marmara University, Faculty of Dentistry, Department of Biochemistry, Istanbul, Turkey

${ }^{3}$ Istanbul University, Cerrahpasa Medical Faculty, Department of Biochemistry, Istanbul, Turkey

Please cite this article as: Ustundag UV, Unal I, Ates PS, Alturfan AA, Yigitbasi T, Emekli Alturfan E. Oxidant-Antioxidant Status and c-myc Expression in BPA- and DEHP-Exposed Zebrafish Embryos. Eur J Biol 2017; 76(1): 26-30.

\begin{abstract}
Di-(2-ethylhexyl)-phthalate (DEHP) is used in polyethylene terephthalate plastics and bisphenol-A (BPA) is used to make polycarbonate plastics. The health-concerning effects of DEHP and BPA indicate their potential toxic effects. The Wnt/ $\beta$ catenin signaling pathway is necessary for embryonic development. The c-MYC proto-oncogene (MYC) has been identified as a Wnt/ $\beta$-catenin target gene, and MYC is a transcription factor that regulates the expression of many gene products related to cell proliferation, growth, differentiation, and apoptosis. Activation and overexpression of the MYC gene has been reported in many human cancers and during tumorigenesis. The aim of this study was to investigate the relationship between oxidantantioxidant balance and c-myc in BPA- and DEHP-exposed zebrafish embryos. The real-time-polymerase chain reaction was used to determine c-myc expression. Lipid peroxidation (LPO), nitric oxide (NO) levels, and superoxide dismutase (SOD) and glutathione-S-transferase (GST) activities were determined in homogenates prepared from pooled embryo samples. Increased expression of c-myc and decreased GST activity were observed in the BPA and DEHP groups. NO levels increased and SOD activity decreased in the BPA group, whereas LPO increased in the DEHP group. Disruption of oxidant-antioxidant balance in DEHP- and BPA-exposed zebrafish embryos was associated with increased c-myc expression and may be an important mechanism for the toxic effects of these chemicals.
\end{abstract}

Keywords: Di(2-ethylhexyl) phthalate, bisphenol A, oxidant-antioxidant balance, c-myc, zebrafish embryo

\section{INTRODUCTION}

Chemicals that produce estrogenic and/or anti-androgenic responses in adult and infant animals are called endocrine disrupting chemicals (EDCs). In recent years, concerns about the potential effects of EDCs have increased; thus, it is important to evaluate their effects in living organisms to understand the metabolic pathways involved (1). Di-(2-ethylhexyl)-phthalate (DEHP) is used in polyethylene terephthalate plastics to increase flexibility and elasticity (2). Phthalates are not chemically bound to plastic materials so they can leach into the environment over time (3). Bisphenol-A (BPA) is used to make polycarbonate plastics, which are found in many products, such as baby bottles, water carboys, and bottles (4). The health-concerning effects of DEHP and BPA indi- cate their potential toxic effects. Widespread exposure to BPA has diverse effects, including impaired reproductive capacity and promotion of obesity and metabolic disease (4-6).

The $\mathrm{Wnt} / \beta$-catenin signaling pathway is important and necessary for embryonic development, and the genes involved in this pathway take part in cell cycle regulation, cell proliferation, differentiation, and apoptosis. Embryonic development may be disrupted when these genes are inhibited. Wnts functions in this pathway (canonical pathway) by disabling the $\beta$-catenin-degradation complex, which leads to APC, Axin, and glycogen synthase kinase 3 beta expression and stabilization of $\beta$-catenin. The stabilized $\beta$-catenin protein translocates to the nucleus and reacts with members of the LEF/TCF transcription factor family 
to induce activation of downstream target genes (7). Aberrant activation of the $\mathrm{Wnt} / \beta$-catenin signaling pathway is a common event in human tumor progression, and mutations in this pathway are generally related to human birth defects, and other diseases (8). Studies related to EDC exposure and the $W n t / \beta$-catenin signaling pathway during the early stages of fetal development are very limited and most have reported developmental delays and childhood cancers (9). MYC is a transcription factor that regulates the expression of many gene products related to cell proliferation, growth, differentiation, and apoptosis. The $c-M Y C$ proto-oncogene (MYC) has been identified as a Wnt/ $\beta$-catenin target gene, and activation and overexpression of the MYC gene has been reported in many human cancers and during tumorigenesis (10).

Oxidative stress is related to the pathogenesis of various disorders. Oxygen radical formation by redox cycling is a critical part of the toxic effects of various compounds when the cell oxidant-antioxidant balance is disrupted. Oxidative stress occurs when reactive oxygen species (ROS) increase, which may affect viability and the severity of inflammation (11).

Zebrafish embryos have been used as alternative models to reduce the number of animal experiments and associated costs within the scope of the "3Rs" principle - replacement, reduction, and refinement of animal experiments. In recent years, zebrafish embryo has gained popularity because of their ease of maintenance, small size, high fecundity, fast development, and optical transparency (12).

The relationship between oxidant-antioxidant balance and c-myc has not been investigated in BPA- and DEHP-exposed zebrafish embryos. Therefore, we evaluated this mechanism in this study.

\section{MATERIAL AND METHODS}

\section{Chemicals Tested}

BPA (CAS no. 80-05-7), DEHP (CAS no 117-81-7), dimethyl sulfoxide (DMSO) (CAS no: 67-68-5), and methanol (CAS no: 67-561) were purchased from Sigma-Aldrich Co. (St Louis, MO, USA). All chemicals were analytical grade and of the highest purity available.

\section{Maintenance of Zebrafish}

Wild type $A B / A B$ strain zebrafish that were originally obtained as a gift from Dr. Fuss of Boğaziçi University (Istanbul, Turkey) were maintained in an apparently disease-free condition in an aquarium rack system (Zebtec, Tecniplast, Italy) at $27 \pm 1^{\circ} \mathrm{C}$ under a light/dark cycle of $14 \mathrm{~L} / 10 \mathrm{D}$. The animals were fed twice daily with commercial flaked fish food supplemented with live Artemia. Reverse osmosis water supplemented with $0.018 \mathrm{mg} \mathrm{L}^{-1}$ Instant Ocean ${ }^{\mathrm{TM}}$ salt was used as the tank water for the experiments. Fertilized embryos were collected and staged by developmental time and morphological criteria as described previously (13). This study was approved by the Committee for Ethics in Animal Experiments of Marmara University.

\section{Embryo Exposure}

After range-finding experiments to identify the lethal concentration that caused $50 \%$ mortality (LC50) of zebrafish embryos, environmentally relevant concentrations of BPA and DEHP that affect development were determined to be $1 \mu \mathrm{g} / \mathrm{L}$ for BPA and $2.5 \mu \mathrm{g} / \mathrm{L}$ for DEHP. Stock solutions of BPA and DEHP were prepared by dissolving each chemical in 0.1 $\mathrm{mL} / \mathrm{L}$ DMSO and methanol, respectively. The embryos were exposed to either DEHP or BPA in well plates for $72 \mathrm{~h}$ post fertilization (hpf). DMSO and methanol were used as the solvent control, whereas the embryo medium was used as the blank control.

\section{Biochemical Analyses}

Zebrafish embryos at $72 \mathrm{hpf}$ were used for biochemical analyses. They were prepared as replicate pools of $72 \mathrm{hpf}$ zebrafish ( $n=5,100$ individuals/pool). A total of 100 embryos were homogenized in $1 \mathrm{~mL}$ PBS for each pool, followed by a brief centrifugation. The supernatant was used to determine the biochemical parameters.

\section{Total Protein Determination}

Total protein level was determined by the Lowry method (14). Briefly, alkaline proteins were reacted with copper ions and reduced by Folin reagent. Absorbance of the product was evaluated at $500 \mathrm{~nm}$ by a spectrophotometer and calculated to express the results of the parameters per protein. Albumin was used as the standard.

\section{Lipid Peroxidation (LPO)}

Malondialdehyde (MDA) level, as an end product of LPO, was determined as thiobarbituric acid reactive substances (TBARS) by the method of Yagi (15). The extinction coefficient of $1.56 \times 10^{5} \mathrm{M}^{-1} \mathrm{~cm}^{-1}$ was used, and LPO was expressed in terms of MDA equivalents as nmol MDA/mg protein.

\section{SOD Activity}

A method based on the ability of SOD to increase the effect of riboflavin-sensitized photo-oxidation of o-dianisidine was used to determine SOD activity in pooled samples. The absorbance of the product was measured at $460 \mathrm{~nm}$ in a spectrophotometer. Net absorbance was calculated by measuring absorbance values at 0 and 8 min of illumination. Results are expressed as $\mathrm{U} / \mathrm{mg}$ protein (16).

\section{NO Determination}

The method was based on the reduction of nitrate to nitrite by vanadium (III) chloride. In acidic media, nitrite and sulfonylamide react with $\mathrm{N}$-(1-naphtyl) ethylenediamine dihydrochloride to form a complex diazonium compound. The colored complex was measured at $540 \mathrm{~nm}$ in a spectrophotometer, and the results are expressed as nmol NO/mg protein (17).

\section{Reverse Transcription (cDNA synthesis) and Quantitative Real-Time Polymerase Chain Reaction}

RNA was isolated from embryos using the Rneasy Mini Kit and Qiacube (Qiagen, Valencia, CA, USA), according to the manufacturer's instructions. Single-stranded cDNA was synthesized 
a

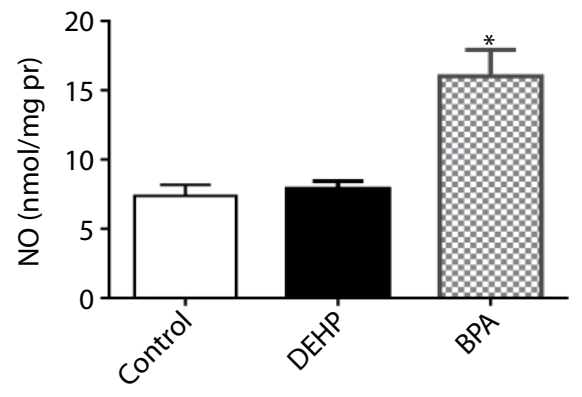

b

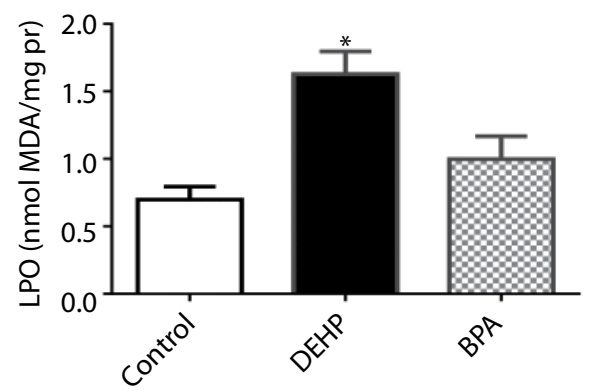

C

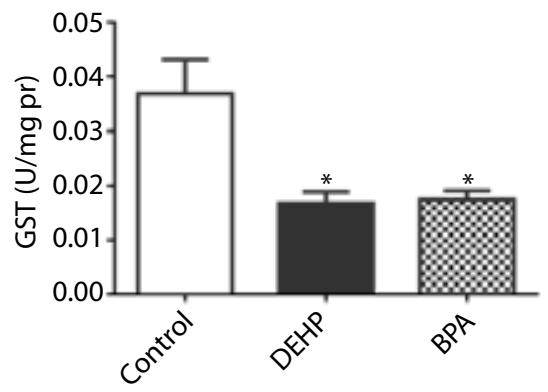

d

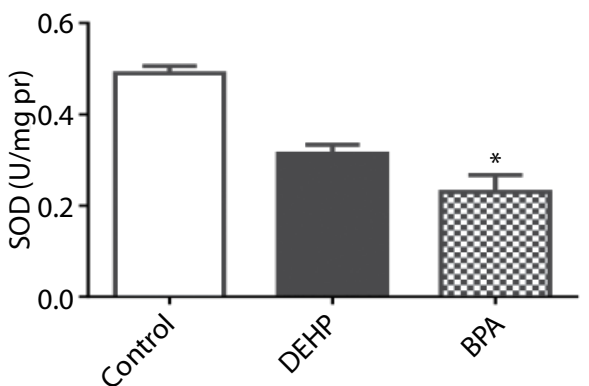

Figure 1. a-d. Results for oxidant-antioxidant parameters. (a) Nitric oxide (NO), (b) lipid peroxidation (LPO), (c) glutathione S-transferase (GST), (d) superoxide dismutase (SOD). Replicate pools of $72 \mathrm{~h}$ post fertilization (hpf) zebrafish ( $\mathrm{n}=5,100$ individuals/pool) were used. Values are given as mean \pm standard deviation.

DEHP: Di (2-ethylhexyl) phthalate; BPA: Bisphenol A

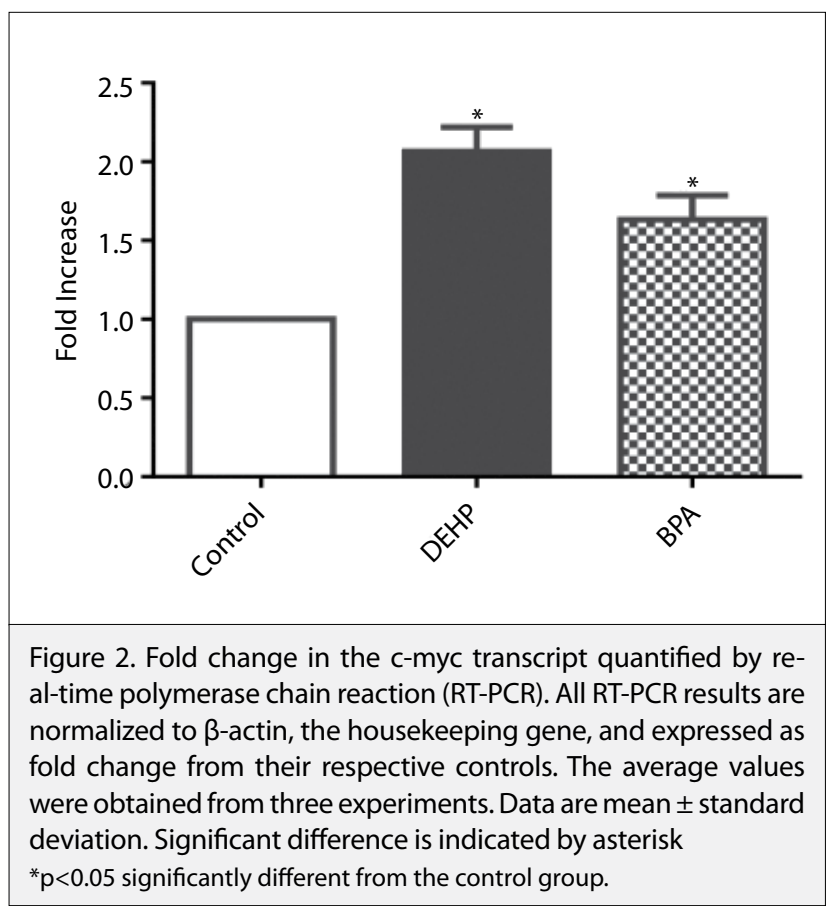

from $1 \mu \mathrm{g}$ of total RNA using $\mathrm{RT}^{2}$ Profiler PCR Arrays (Qiagen). PCRs were performed using the DNA Master SYBR Green kit (Qiagen). The expression of c-myc (forward primer, 5'-TGACTGTGGAAAAGCGACAG-3' reverse primer, 5'-GCTGCTGTTGATGCTGTGAT-3' was evaluated by quantitative RT-PCR using the Qiagen Rotor Gene-Q Light Cycler instrument. $\beta$-actin (forward primer: 5'-GCTCACCATGGATGATGATATCGC-3'reverse primer: 5'-GGAGGAGCAATGATCTTGATCTTC-3') was used as the housekeeping gene (18). Relative transcript levels were calculated using the DDCT method by normalizing the values to the housekeeping gene (19).

\section{Statistical Analysis}

One-way analysis of variance with a post-hoc Dunn's multiple comparison test were used to analyze the differences between the groups using Graph Pad 6 software (GraphPad Software, La Jolla, CA, USA). A $p$-value $\leq 0.05$ was considered significant.

\section{RESULTS}

The data analysis did not indicate any difference between the solvent control and blank control for the indices investigated in this study; therefore, the blank control test data are provided in the following sections.

\section{Biochemical analyses}

Results of the biochemical analyses are given in Figure 1. NO levels increased significantly and GST and SOD activities decreased significantly in the BPA group compared with those in the control group $(p<0.05)$. LPO increased significantly and GST activity decreased significantly in the DEHP group compared with those in the control group $(p<0.05)$. 


\section{RT-PCR analysis}

c-myc expression levels are given as fold changes in transcripts quantified by RT-PCR. The RT-PCR results were normalized to $\beta$-actin and expressed as the change from their respective controls. The average values obtained from three experiments are given in Figure 2. Increased expression of c-myc was observed compared with the control in both groups $(p<0.05)$.

\section{DISCUSSION}

Although previous studies have shown that EDCs exert their effects by interfering with homeostasis, reproduction, and development, it has recently been shown that the mechanisms are much broader than originally recognized (20). Accordingly, we showed that DEHP disturbed the oxidant-antioxidant balance by increasing LPO and decreasing GST activity in developing zebrafish embryos. We also showed that BPA increased NO and decreased GST and SOD activities.

A balance between reduction and oxidation (redox) of chemicals must be maintained for cellular development, growth, and survival. ROS are generated during normal cellular metabolism, such as superoxide anions, peroxides, and hydroxyl radicals, and cells have developed antioxidant mechanisms to regulate and scavenge excess ROS to prevent deleterious effects. If this balance is disrupted by environmental toxicants or EDCs, the same ROS that were beneficial to the cell can cause mutations, unchecked cell growth, and impair cellular signals $(21,22)$. Moreover, increased oxidative stress is related to aging, cardiovascular disease, neuronal degeneration, and the development and progression of cancers (22).

Due to increasing concern about the risk of EDC exposure, more studies have examined the underlying mechanisms over the past 10 years. For instance, there is growing evidence showing that the induction of ROS by BPA may contribute significantly to its toxicity and carcinogenic potential (21).

However, there are conflicting findings for the pro-oxidant and antioxidant roles of BPA. Differences in doses, durations, and cellular microenvironments used in different studies may be influencing the pro-oxidant/antioxidant role of BPA. Many in vivo and in vitro studies support the pro-oxidant actions of BPA, while the antioxidant role has only been reported in vitro. The structure of BPA may promote its potential to act as a weak antioxidant via electron loss through the $\mathrm{O}-\mathrm{H}$ bond (23). On the other hand, the reduction in ROS after BPA exposure indicates the ROS scavenging potential of BPA and changes in the cellular microenvironment, cell type, and cell signaling responses contribute to the observed reduction in oxidative stress $(24,21)$.

DEHP treatment induces oxidative stress in rat kidneys, as evidenced by significant decreases in glutathione peroxidase and SOD activities as well as GSH levels along with a marked decrease in thiol content and an increase in TBARS level (25).

Consistent with these studies, DEHP-exposed zebrafish embryos had significantly increased MDA levels, and BPA-exposed ze- brafish embryos had significantly increased NO levels and decreased antioxidant enzyme activities. Accordingly, Kuan et al. (26) suggested that BPA-glycidyl-methacrylate can induce NO, ROS, and inflammatory cytokine expression by macrophages.

In our study, BPA and DEHP exposure increased c-myc expression in zebrafish embryos. Pfeifer et al. (27) reported that BPA upregulates the expression of c-Myc and other crucial oncogenic cell-cycle regulatory proteins as well as induces ROS in mammary gland cells. Apart from the well-established involvement of c-Myc in regulation of the cell cycle, differentiation, and apoptosis, further functions of c-Myc include amino acid and nucleotide synthesis, regulation of lipid metabolism, glycolysis, and mitochondrial homeostasis. c-Myc has also been suggested to function in determining cellular redox balance. Benassi et al. (28) reported that c-Myc phosphorylation-dependent activation of the GSH-directed survival pathway contributes to resistance to oxidative stress in tumor cells, which generally exhibit dysregulated c-Myc expression. Our study showed increased c-myc expression and oxidative stress in the EDC-exposed groups. Therefore, increased c-myc expression might be due to the protective mechanism of the embryo to improve the oxidant-antioxidant balance that has been disrupted by BPA or DEHP. Given these results, further studies are needed to investigate the mechanisms of the toxic effects of BPA and DEHP. More quantitative results are needed regarding the crosstalk between oxidative stress and c-myc.

\section{REFERENCES}

1. Kincha $C D$, Ibhazehiebo $K$, Jeong JH, Habibia HR, Kurrasch DM. Low-dose exposure to bisphenol A and replacement bisphenol $\mathrm{S}$ induces precocious hypothalamic neurogenesis in embryonic zebrafish. Proc Nat Acad Sci 2015; 112(5): 1475-80. [CrossRef]

2. Wan HT, Leung PY, Zhao YG, Wei X, Wong MH, Wong CK. Blood plasma concentrations of endocrine disrupting chemicals in Hong Kong populations. J Hazard Mater 2013; 261: 763-9. [CrossRef]

3. Uren-Webster TM, Lewis C, Filby A, Paull GC, Santos EM. Mechanisms of toxicity of di(2-ethylhexyl) phthalate on the reproductive health of male zebrafish. Aquat Toxicol 2010; 99(3): 360-9. [CrossRef]

4. Akyüz S, Yarat A, Egil E. Dental materials containing bisphenol-A: current approach. Clin Exp Health Sci 2011; 1(3): 190-5.

5. Alonso-Magdalena P, Vieira E, Soriano S, Menes L, Burks D, Quesada $\mathrm{I}$, et al. Bisphenol A exposure during pregnancy disrupts glucose homeostasis in mothers and adult male offspring. Environ Health Perspect 2010; 118(9): 1243-50. [CrossRef]

6. Adewale HB, Todd KL, Mickens JA, Patisaul HB. The impact of neonatal bisphenol-A exposure on sexually dimorphic hypothalamic nuclei in the female rat. Neurotoxicology 2011; 32(1): 38-49. [CrossRef]

7. Huang $\mathrm{H}, \mathrm{He} X$. Wnt/beta-catenin signaling: new (and old) players and new insights. Curr Opin Cell Biol 2008; 20(2): 119-25. [CrossRef]

8. Logan $\mathrm{CY}$, Nusse R. The Wnt signaling pathway in development and disease. Annu Rev Cell Dev Biol 2004; 20: 781-810. [CrossRef]

9. Stuppia L, Franzago, Ballerini P, Gatta V, Antonucci I. Epigenetics and male reproduction: the consequences of paternal lifestyle on fertility, embryo development, and children lifetime health. Clin Epigenetics 2015; 7: 120. [CrossRef] 
10. Casey SC, Tong L, Li Y, Do R, Walz S, Fitzgerald KN, et al. MYC Regulates the anti-Tumor immune response through CD47 and PD-L1. Science 2016; 352(6282): 227-31. [CrossRef]

11. Simonian NA, Coyle JT. Oxidative stress in neurodegenerative diseases. Annu Rev Pharmacol Toxicol 1996; 36: 83-106. [CrossRef]

12. Mudbhary R, Sadler KC. Epigenetics, development, and cancer: zebrafish make their mark. Birth Defects Res C Embryo Today 2011; 93(2): 194-203. [CrossRef]

13. Westerfield M. The Zebrafish Book, A Guide for the Laboratory Use of Zebrafish. University of Oregon Press, Oregon, USA (1995).

14. Lowry OH, Rosebrough NJ, Farr AL, Randall RJ. Protein measurement with the Folin phenol reagent. J Biol Chem 1951; 193(1): 26575.

15. Yagi K. Assay for blood plasma or serum. Methods Enzymol 1981; 105: 328-37. [CrossRef]

16. Mylorie AA, Collins H, Umbles C, Kyle J. Erythrocyte SOD activity and other parameters of copper status in rats ingesting lead acetate. Toxicol Appl Pharmacol 1986; 82(3): 512-20. [CrossRef]

17. Miranda KM, Espey MG, Wink DA. A rapid, simple spectrophotometric method for simultaneous detection of nitrate and nitrite. Nitric Oxide 2001; 5(1): 62-71. [CrossRef]

18. Hui SP, Sengupta D, Lee SGP, Sen T, Kundu S, Mathavan S. Genome Wide Expression Profiling during Spinal Cord Regeneration Identifies Comprehensive Cellular Responses in Zebrafish. PLoS One 2014; 9(1): e84212. [CrossRef]

19. Livak KJ, Schmittgen TD. Analysis of relative gene expression data using real-time quantitative PCR and the 2(-Delta Delta C(T)) Method. Methods 2001; 25(4): 402-8. [CrossRef]

20. Diamanti-Kandarakis E, Bourguignon J, Giudice LC, Hauser R, Prins GS, Soto AM, et al. Endocrine-disrupting chemicals: an Endocrine
Society scientific statement. Endocr Rev 2009; 30(4): 293-342. [CrossRef]

21. Gassman NR. Induction of oxidative stress by bisphenol $A$ and its pleiotropic effects. Environ Mol Mutagen 2017; 58(2): 60-71. [CrossRef]

22. Liou GY, Storz P. Reactive oxygen species in cancer. Free Radic Res 2010; 44(5): 479-96. [CrossRef]

23. Chepelev NL, Enikanolaiye MI, Chepelev LL, Almohaisen A, Chen Q, Scoggan KA, et al. Bisphenol A activates the Nrf1/2-antioxidant response element pathway in HEK 293 cells. Chem Res Toxicol 2013; 26(3): 498-506. [CrossRef]

24. Ponniah M, Billett EE, De Girolamo LA. Bisphenol A increases BeWo trophoblast survival in stress-induced paradigms through regulation of oxidative stress and apoptosis. Chem Res Toxicol 2015; 28(9): 1693-703. [CrossRef]

25. Erkekoglu P, Giray BK, Kizilgun M, Rachidi W, Hininger-Favier I, Roussel AM, et al. Di(2-ethylhexyl)phthalate-induced renal oxidative stress in rats and protective effect of selenium. Toxicol Mech Methods 2012; 22(6): 415-23. [CrossRef]

26. Kuan YH, Huang FM, Li YC, Chang YC. Proinflammatory activation of macrophages by bisphenol A-glycidyl-methacrylate involved NFKB activation via PI3K/Akt pathway. Food Chem Toxicol 2012; 50(11): 4003-9. [CrossRef]

27. Pfeifer D, Chung YM, Hu MC. Efects of low-dose bisphenol A on DNA damage and proliferation of breast cells: the role of c-Myc. Environ Health Perspect 2015; 123(12): 1271-9. [CrossRef]

28. Benassi B, Fanciulli M, Fiorentino F, Porrello A, Chiorino G, Loda M, et al. c-Myc phosphorylation is required for cellular response to oxidative stress. Mol Cell 2006; 21(4): 509-19. [CrossRef] 